\title{
Improved patient satisfaction using ingenol mebutate gel $0.015 \%$ for the treatment of facial actinic keratoses: a prospective pilot study
}

This article was published in the following Dove Press journal:

Clinical, Cosmetic and Investigational Dermatology

20 April 2016

Number of times this article has been viewed

\author{
Joanna Emilio' \\ Michelle Schwartz ${ }^{2-4}$ \\ Eleanor Feldman ${ }^{2-4}$ \\ Amy Kalowitz Bieber ${ }^{2-4}$ \\ Amanda Bienenfeld ${ }^{2-4}$ \\ Min-Kyung Jung' \\ Daniel M Siegel ${ }^{2,3}$ \\ Orit Markowitz ${ }^{2-4}$ \\ 'Department of Dermatology, NYIT \\ College of Osteopathic Medicine, \\ Old Westbury, ${ }^{2}$ Department of \\ Dermatology, SUNY Downstate \\ Medical Center, ${ }^{3}$ Department of \\ Dermatology, NY Harbor Healthcare \\ System, Brooklyn, ${ }^{4}$ Department of \\ Dermatology, Mount Sinai Medical \\ Center, New York, NY, USA
}

Correspondence: Orit Markowitz Department of Dermatology, Mount Sinai Medical Center, 5 East 98th Street, 5th

Floor, New York, NY 10029, USA

Tel + | 2 I $224 \mid 9728$

Fax + I 212978 II97

Email omarkowitz@gmail.com

\begin{abstract}
Actinic keratoses (AKs), especially on areas of the face, have a negative impact on a patient's quality of life (QoL). These lesions manifest on sun-damaged skin and have the potential to progress to squamous cell carcinoma. Field-directed therapy alone and in combination with lesion-directed treatment is effective in clearing both visible and nonvisible AK lesions. Topical treatments of AKs thus have the potential to improve a patient's well-being. However, evidence demonstrating improvements in patient QoL is limited, and is mostly based on observational or retrospective studies. Some prospective studies have reported unchanged or even worsening QoL despite excellent treatment outcomes. Our prospective, pilot study demonstrated a significant increase in QoL in 28 subjects with AKs of the face treated with ingenol mebutate gel $0.015 \%$. QoL was assessed at days 0 and 60 using the Skindex-16 survey. Mean overall scores improved from $24.5 \%$ at baseline to $15.5 \%$ at day $60(P=0.031)$. Improvements in QoL were consistent with an $80 \%$ reduction in AK lesion number at day 60 . These improved QoL findings are in line with those from a recent retrospective study using ingenol mebutate $0.015 \%$ gel. This study therefore further demonstrates the potential for field therapy to improve both treatment outcomes and patient satisfaction.
\end{abstract}

Keywords: actinic keratosis, squamous cell carcinoma, ingenol mebutate gel, local skin reaction, quality of life, Skindex-16

\section{Introduction}

Actinic keratosis (AK) results primarily from the damaging effects of long-term ultraviolet radiation on the skin. ${ }^{1-3}$ Accordingly, the majority of lesions appear on the head, neck, and upper limbs. ${ }^{4,5}$ Considered a field disease, AK typically involves the presence of subclinical and therefore nonvisible lesions within an area of damaged skin, in close proximity to the clinically detectable AK lesion. ${ }^{6,7}$ In the United States, clinically apparent lesions are generally treated individually with cryotherapy. ${ }^{8}$ However, topical therapies can potentially treat an entire field of damaged skin, including not only the visible lesions but also the subclinical lesions as well. Recommended topical therapies for field-directed treatment of AKs include diclofenac gel, 5-fluorouracil (5-FU) cream, imiquimod cream, and ingenol mebutate gel. ${ }^{9}$ Photodynamic therapy with red, blue, or daylight illumination can also be used as a field-directed or lesion-directed therapeutic approach for AK. ${ }^{10}$

One difficulty in assessing AK treatment success is that the criteria for success in dermatologic disease are not easily quantifiable. Factors such as the patient's quality of life (QoL) as well as the clinical outcome determine treatment success. ${ }^{11}$ In dermatologic conditions, variables that impact QoL are often highly context dependent, 
such as the location of the damaged skin or the age and sex of the patient. ${ }^{12-14}$ In addition, characteristics of the treatment regimen itself, such as its length, complexity, or association with adverse events, are likely to affect patient QoL. Taken together, these factors make it difficult to predict how a particular treatment regimen might affect a patient's QoL, even with demonstration of clinical improvement.

As such, QoL may be based more on a balance between treatment tolerance and treatment outcomes. Some prior studies have demonstrated unchanged or even decreased QoL despite excellent treatment outcomes. ${ }^{15,16}$ Recently, a post hoc, exploratory analysis of AK subjects enrolled in Phase 3 clinical trials involving ingenol mebutate gel found that this therapy was associated with significant improvements in QoL. ${ }^{17}$ Here, we report the results of QoL assessments from a prospective clinical study in subjects with AKs of the face who were treated with ingenol mebutate gel $0.015 \%$ for three consecutive days.

\section{Methods}

This study was part of a clinical trial that examined the effects of ingenol mebutate gel $0.015 \%$ applied once daily for three consecutive days to 28 subjects with AKs of the face. Treatment effects were assessed by clinical assessment, noninvasive imaging, and a QoL questionnaire. Subjects were recruited from the Brooklyn Campus of the Veterans Affairs New York Harbor Healthcare System, all were white males, $\geq 65$ years of age, who had at least seven AKs on the face. Patients provided written informed consent and allowed photographs of the selected treatment area to be taken. This study received continuing Institutional Review Board approval on March 13, 2015 from the New York Harbor Healthcare System Department of Veterans Affairs' Subcommittee for Human Studies.

\section{Subject treatment and assessment}

Ingenol mebutate gel $0.015 \%$ was applied by the clinical staff once daily for three consecutive days to a $25 \mathrm{~cm}^{2}$ contiguous area of the face that contained at least three clinical AKs. Local skin reactions (LSRs) were recorded with clinical photographs taken on each day of treatment. The efficacy of ingenol mebutate gel $0.015 \%$ was assessed at day 60 by clinical assessment and by the Skindex-16 dermatologic questionnaire, a single-page, 16-item patient questionnaire that measures the effects of skin disease on the subject's QoL (Table 1). ${ }^{18}$ The 16 questions cover three QoL subscales: symptoms (Q1-Q4), emotions (Q5-Q11), and functioning (Q12-Q16). Responses are recorded using a 0-6 numerical
Table I Summary of Skindex-16 survey questions

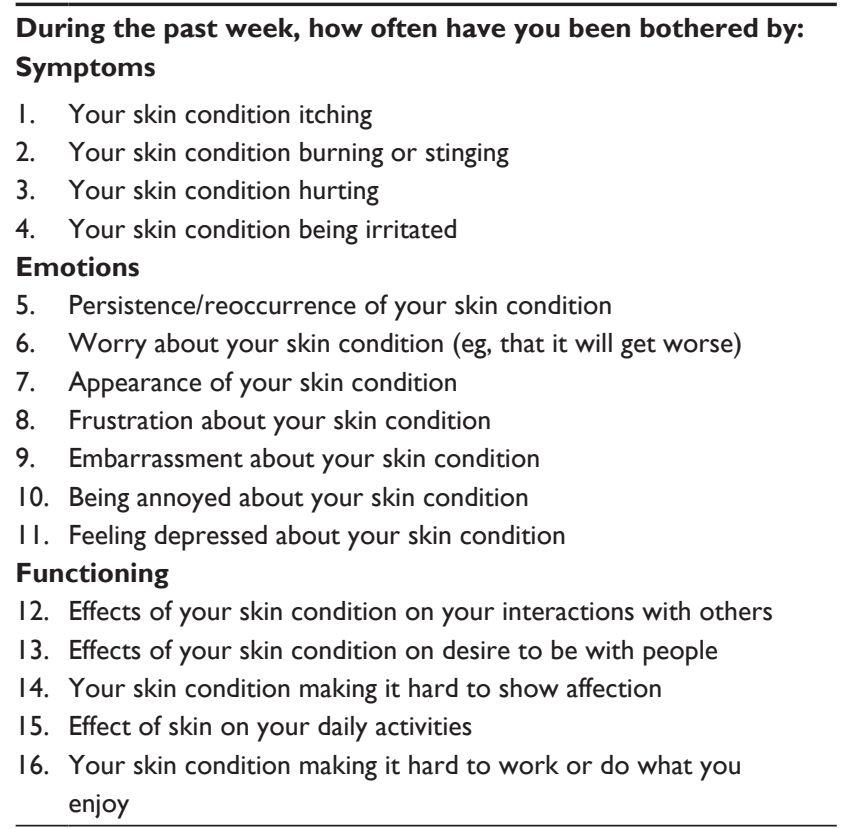

Notes: Subjects were asked 16 questions that covered three domains. Response choices for all questions were indicated on a continuous, numeric analog scale, where 0 indicates "never bothered" and 6 indicates "always bothered".

analog scale to measure bothersomeness, where 0 indicates "never bothered" and 6 indicates "always bothered."

\section{Statistical methods}

Overall QoL scores and individual subscale QoL scores were computed as a standardized percentage by adding the scores from all questions, dividing this number by the maximum possible score, and then multiplying by 100 . Means and standard deviations were computed for QoL scores, and a paired $t$-test was performed to test the null hypothesis that the mean QoL score did not change from days 0 to 60 . Statistical significance was determined by alpha $=0.05$, with no multiplecomparison correction in order to maximize statistical power. For analysis of the potential relationship between the subject's LSRs and QoL improvement, only subjects who completed the Skindex-16 survey on both days 0 and 60 were included in the analysis. The value of Cohen's $d$ was computed as a measure of effect size. The practical significance of the effect size was based on the value of Cohen's $d$, using the guidelines of $d=0.2$ (small), $d=0.5$ (medium), and $d=0.8$ (large).

\section{Results}

\section{Relationship between clinical assessment and subjects' QoL}

Of the 28 subjects enrolled in the study, 25 subjects completed the Skindex-16 survey on both days 0 and 60 and were included in the analysis. The mean (standard deviation 
[SD]) overall Skindex-16 score in the 25 subjects was 24.5 (20.9) at day 0 and 15.5 (19.3) at day 60 of the study (Figure 1A). The reduction in Skindex-16 scores indicated a statistically significant improvement in QoL $(P=0.031)$. In addition, there were numeric reductions in scores in all three subscales (symptoms, emotions, and functioning), with symptoms reducing from 24.3 (20.6) to 16.3 (18.7), emotions reducing from 34.1 (26.6) to 20.2 (23.8), and functioning reducing from 13.5 (22.1) to 8.3 (18.4). However, statistical significance was achieved in the emotions subscale only $(P=0.011)$. Improvements in QoL were consistent with the clinical assessment, in which a $77 \%$ reduction in lesions $(95 \%$ CI, $68 \%-86 \%$ ) was recorded. Specifically, in areas of the skin treated with ingenol mebutate gel $0.015 \%, 83$ lesions (sum of clinical and subclinical) were identified at baseline, and 19 lesions were identified at day 60 (Figure 1B).

\section{Impact of LSRs on subjects' QoL}

Following application of ingenol mebutate gel $0.015 \%$, LSRs peaked at day 3 in the 3-day treatment regimen (Figure 2). Responses were classified as mild, moderate, or severe, with most subjects $(75 \%)$ having a mild-to-moderate response. Skindex-16 scores were then grouped by LSR severity for subjects with mild $(\mathrm{n}=9)$, moderate $(\mathrm{n}=11)$, and severe $(\mathrm{n}=5)$ reactions. Numeric improvements were seen in overall QoL scores regardless of LSR severity, although the differences did not reach statistical significance (Figure 3 ). The potential impact of ingenol mebutate treatment on QoL was further examined by calculating the effect size using Cohen's $d$. For overall QoL scores, the absolute Cohen's $d$ values were 2.1, 1.8 , and 0.2 for subjects with mild, moderate, and severe LSRs, respectively (Figure 3). Thus, ingenol mebutate gel $0.015 \%$ had a large positive impact on QoL for subjects with mild and moderate LSRs and little impact on subjects with severe LSRs.

\section{Discussion}

Studies on the relationship between the use of AK topical therapies and QoL have yielded inconsistent results despite the demonstrated clinical effectiveness of these treatments. In the VA Topical Tretinoin Chemoprevention (VATTC) Trial, past use of 5-FU was associated with a worse QoL. ${ }^{16}$ However, this study did not track a single population of subjects before and after 5-FU use, thus limiting the interpretation of this result. Health-related QoL was investigated in a study of 118 AK subjects treated with imiquimod $0.05 \%$ cream for 8 weeks, in which QoL was assessed using a Skindex-16 survey and the Skin Cancer Index..$^{15}$ The small improvements in QoL seen at week 8 were judged to be lacking in clinical relevance. Low baseline impairments in QoL may have limited the ability to detect more robust improvements. A study of AK subjects treated with 3\% diclofenac in 2.5\% hyaluronic acid gel for up to 6 months demonstrated improvements in both clinical outcomes and QoL, as assessed by the
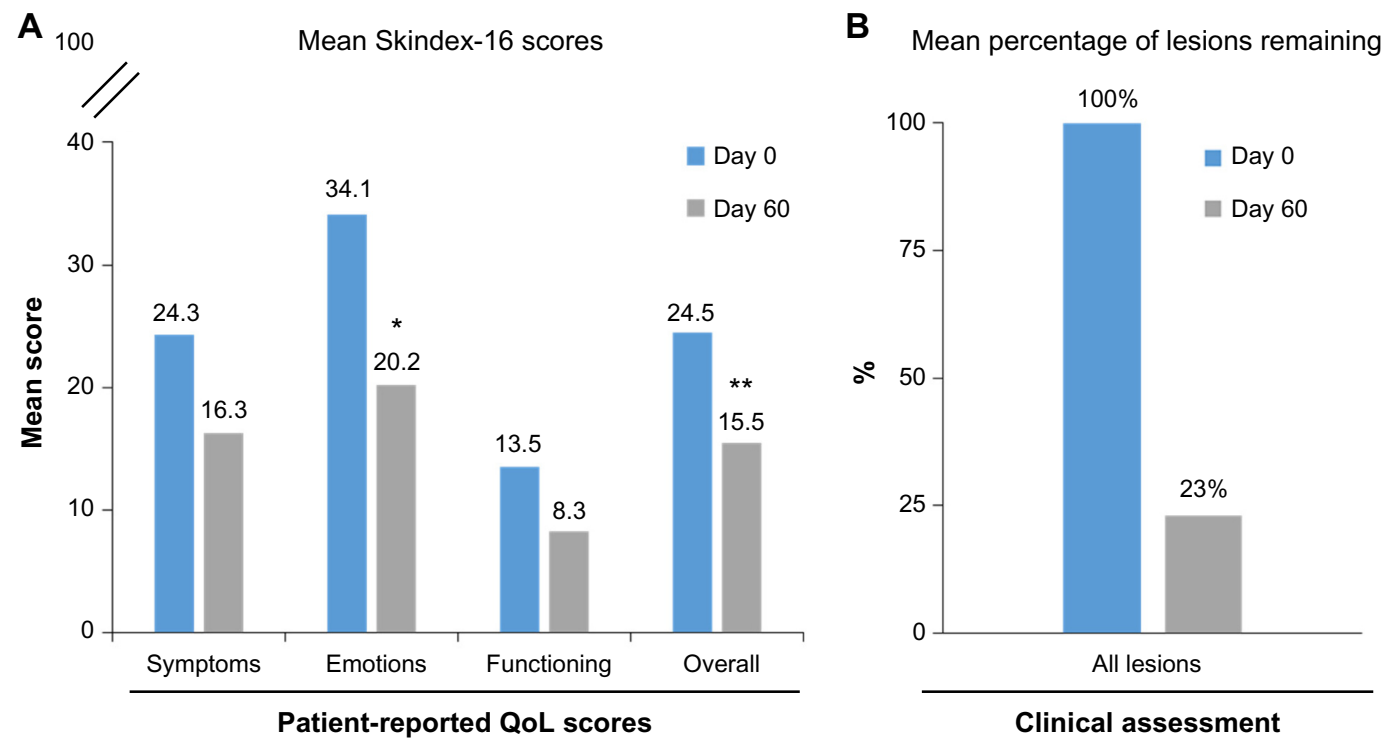

Clinical assessment

Figure I QoL and clinical assessments of ingenol mebutate treatment effect

Notes: (A) Mean QoL Skindex-16 scores. Mean scores for days 0 and 60 are shown for each subscale and the overall score. Differences were significant for the emotions subscale and the overall score; $* P=0.01$ I, $* * P=0.031$. (B) Lesion counts. In areas of the skin treated with ingenol mebutate gel $0.015 \%, 83$ lesions (sum of clinical and subclinical) were identified at baseline, and 19 lesions were identified at day 60 , corresponding to a $77 \%$ lesion clearance rate $(95 \% \mathrm{Cl}, 68 \%-86 \%)$.

Abbreviations: $\mathrm{Cl}$, confidence interval; QoL, quality of life. 


\section{Day 1}
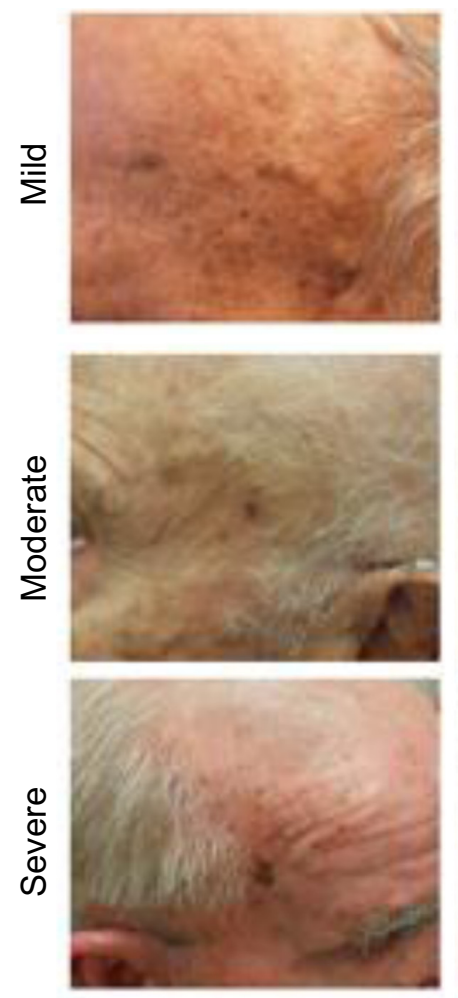

Day 2
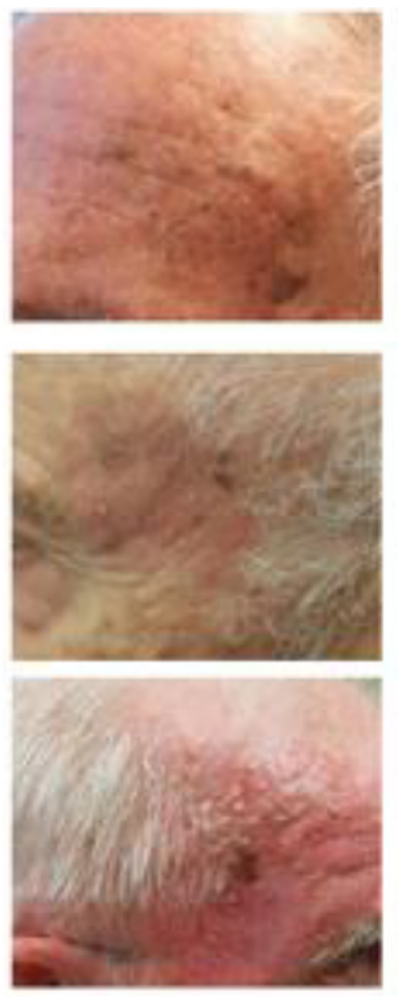

Day 3
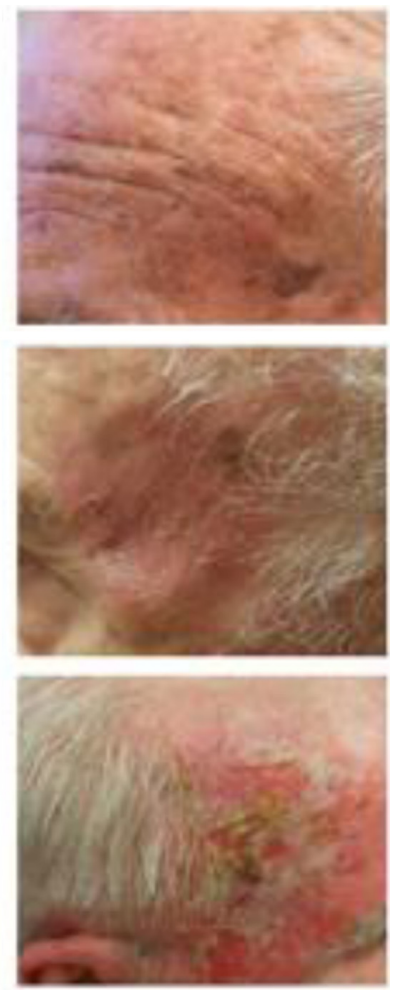

Figure 2 Clinical photographs of representative mild, moderate, and severe LSRs at days I, 2, and 3 following ingenol mebutate treatment. Notes: At day 3, the number of subjects with mild, moderate, and severe LSRs was 9, 12, and 7, respectively. Abbreviation: LSR, local skin reaction.

Dermatology Quality of Life Index, despite the fact that skin reactions led to interruption or discontinuation of treatment in $13.6 \%$ of the subjects. ${ }^{19}$ Finally, a post hoc analysis of 1,005 subjects enrolled in Phase 3 ingenol mebutate clinical trials for the treatment of AK revealed improved QoL, as assessed by the Treatment Satisfaction Questionnaire for Medication and Skindex-16 surveys. ${ }^{17}$ For the Skindex-16 survey of subjects treated with ingenol mebutate on the face and scalp, significant improvements from baseline versus vehicle were seen in the overall score as well as in the scores for the three subscales, with the emotions subscale showing greatest improvement in mean score, followed second by symptoms and then functioning.

In contrast to the post hoc analysis of the Phase 3 ingenol mebutate study, our present study was a prospective analysis of 28 subjects at a single center. Despite this relatively small sample size, we saw a significant improvement in overall QoL as measured by the Skindex-16 survey. Interestingly, and consistent with the findings of the post hoc analysis, the greatest numeric improvement was seen in the emotions subscale, followed by the symptoms and then the functioning subscales.

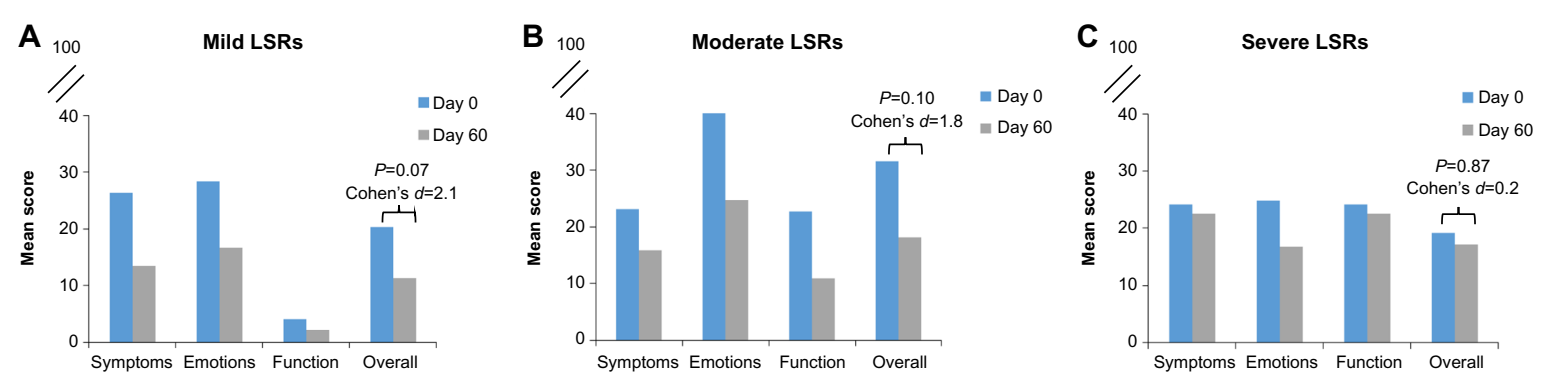

Figure 3 Mean subscale and overall Skindex-16 scores for subjects with (A) mild $(n=9),($ B) moderate $(n=I I)$, and $(\mathbf{C})$ severe $(n=5)$ LSRs.

Note: Only subjects who completed the survey on days 0 and 60 were included in the analysis.

Abbreviation: LSR, local skin reaction. 
As with other topical AK treatments, LSRs occur with the use of ingenol mebutate gel $0.015 \%$ and have the potential to negatively affect QoL. When the subjects in our study were grouped by the severity of their LSRs, each of the groups attained numeric improvements in overall QoL. The small cohort sizes may have precluded the attainment of statistical significance. Calculation of effect size using Cohen's $d$ revealed that subjects with mild and moderate LSRs achieved a large improvement in overall QoL scores, while subjects with severe LSRs did not have an improvement in QoL. Nevertheless, the AK clearance rates in subjects who experienced severe LSRs were similar to those in subjects with mild and moderate LSRs. These observations suggest that patients who experience severe LSRs early in the treatment course might have limited improvements in QoL even if they show significant clearance of AK lesions. These patients might benefit from additional counseling that emphasizes not only their achievement of clinical improvement but also the importance of detecting and treating new lesions that might arise in the future.

Limitations of this study include the relatively small cohort size, the homogeneity of the subjects with respect to race, age, and sex, and the fact that the study was performed at a single center.

In summary, our prospective clinical study demonstrated improved QoL in subjects who were treated with ingenol mebutate gel $0.015 \%$ for AKs. Improvements in QoL were consistent with the clinical improvements seen in these subjects. These results confirm reports of treatment-related QoL improvements in AK patients.

\section{Acknowledgment}

Editorial support was provided by $p$-value communications, and funded by LEO Pharma Inc.

\section{Disclosure}

The authors report no conflicts of interest in this work.

\section{References}

1. Wheller L, Soyer HP. Clinical features of actinic keratoses and early squamous cell carcinoma. Curr Probl Dermatol. 2015;46:58-63.
2. Berman B, Cockerell CJ. Pathobiology of actinic keratosis: ultravioletdependent keratinocyte proliferation. J Am Acad Dermatol. 2013; 68(1 Suppl 1):S10-S19.

3. Dodds A, Chia A, Shumack S. Actinic keratosis: rationale and management. Dermatol Ther (Heidelb). 2014;4(1):11-31.

4. Frost CA, Green AC. Epidemiology of solar keratoses. Br J Dermatol. 1994;131(4):455-464.

5. Salasche SJ. Epidemiology of actinic keratoses and squamous cell carcinoma. J Am Acad Dermatol. 2000;42(1 Pt 2):4-7.

6. Slaughter DP, Southwick HW, Smejkal W. Field cancerization in oral stratified squamous epithelium: clinical implications of multicentric origin. Cancer. 1953;6(5):963-968.

7. Goldenberg G, Perl M. Actinic keratosis: update on field therapy. J Clin Aesthet Dermatol. 2014;7(10):28-31.

8. Hagele TJ, Levender MM, Davis SA, Williford PM, Feldman SR. Practice trends in the treatment of actinic keratosis in the United States: $0.5 \%$ fluorouracil and combination cryotherapy plus fluorouracil are underused despite evidence of benefit. J Cutan Med Surg. 2012;16:107-114.

9. Stockfleth E. The paradigm shift in treating actinic keratosis: a comprehensive strategy. J Drugs Dermatol. 2012;11(12):1462-1467.

10. Wiegell SR. Update on photodynamic treatment for actinic keratosis. Curr Probl Dermatol. 2015;46:122-128.

11. Chren MM. The Skindex instruments to measure the effects of skin disease on quality of life. Dermatol Clin. 2012;30(2):231-236.

12. Esmann S, Vinding GR, Christensen KB, Jemec GB. Assessing the influence of actinic keratosis on patients' quality of life: the AKQoL questionnaire. Br J Dermatol. 2013;168(2):277-283.

13. de Jager ME, de Jong EM, van de Kerkhof PC, Evers AW, Seyger MM. An intrapatient comparison of quality of life in psoriasis in childhood and adulthood. J Eur Acad Dermatol Venereol. 2011;25(7):828-831.

14. Mabuchi T, Yamaoka H, Kojima T, Ikoma N, Akasaka E, Ozawa A. Psoriasis affects patient's quality of life more seriously in female than in male in Japan. Tokai J Exp Clin Med. 2012;37(3):84-88.

15. Waalboer-Spuij R, Holterhues C, van Hattem S, et al. Patient perception of imiquimod treatment for actinic keratosis and superficial basal cell carcinoma in 202 patients. Dermatology. 2015;231:56-62.

16. Weinstock MA, Lee KC, Chren MM, Marcolivio K; VATTC Trial Group. Quality of life in the actinic neoplasia syndrome: the VA Topical Tretinoin Chemoprevention (VATTC) Trial. J Am Acad Dermatol. 2009;61(2):207-215.

17. Augustin M, Tu JH, Knudsen KM, Erntoft S, Larsson T, Hanke CW. Ingenol mebutate gel for actinic keratosis: the link between quality of life, treatment satisfaction, and clinical outcomes. JAm Acad Dermatol. 2015;72(5):816-821.

18. Chren MM, Lasek RJ, Sahay AP, Sands LP. Measurement properties of Skindex-16: a brief quality-of-life measure for patients with skin diseases. J Cutan Med Surg. 2001;5(2):105-110.

19. Pflugfelder A, Welter AK, Leiter U, et al. Open label randomized study comparing 3 months vs. 6 months treatment of actinic keratoses with $3 \%$ diclofenac in $2.5 \%$ hyaluronic acid gel: a trial of the German Dermatologic Cooperative Oncology Group. J Eur Acad Dermatol Venereol. 2012;26(1):48-53.
Clinical, Cosmetic and Investigational Dermatology

\section{Publish your work in this journal}

Clinical, Cosmetic and Investigational Dermatology is an international, peer-reviewed, open access, online journal that focuses on the latest clinical and experimental research in all aspects of skin disease and cosmetic interventions. All areas of dermatology will be covered; contributions will be welcomed from all clinicians and

\section{Dovepress}

basic science researchers globally. This journal is indexed on CAS. The manuscript management system is completely online and includes a very quick and fair peer-review system, which is all easy to use. Visit http://www.dovepress.com/testimonials.php to read real quotes from published authors. 